\title{
Antibiotic accumulation, growth performance, physiologic status and intestinal flora diversification of Nile tilapia (Oreochromis niloticus) feed by diets supplemented with different dose of sulfamethoxazole
}

\section{Longxiang Fang}

Chinese Academy of Fishery Sciences Freshwater Fisheries Research Center

Xi Chen

Chinese Academy of Fishery Sciences Freshwater Fisheries Research Center

Xiangbao Shan

Nanjing Agricultural University

Liping Qiu

Chinese Academy of Fishery Sciences Freshwater Fisheries Research Center

\section{Limin Fan}

Chinese Academy of Fishery Sciences Freshwater Fisheries Research Center

\section{Shunlong Meng}

Chinese Academy of Fishery Sciences Freshwater Fisheries Research Center

Chao Song ( $\nabla$ songc@ffrc.cn )

Nanjing Agricultural University

\section{Research}

Keywords: Sulfamethoxazole, Nile tilapia, Antibiotic accumulation, Growth performance, Physiologic status

Posted Date: January 20th, 2021

DOl: https://doi.org/10.21203/rs.3.rs-150011/v1

License: (c) (1) This work is licensed under a Creative Commons Attribution 4.0 International License.

Read Full License 


\section{Abstract \\ Background}

Sulfamethoxazole (SMZ) is an antibiotic used globally to treat fish disease in aquaculture, but the effects of exposure to legal aquaculture doses of SMZ in fish are still unclear. To comprehensively investigate the effects of exposure to legal doses of sulfamethoxazole (SMZ) in Nile tilapia (Oreochromis niloticus), fish were exposed to diets supplemented with different doses of SMZ (blank group, normal feed; LS, $0.67 \mathrm{~g} / \mathrm{kg} ; \mathrm{MS}, 6.67 \mathrm{~g} / \mathrm{kg}$ and HS, $33.33 \mathrm{~g} / \mathrm{kg}$ ) for 4 weeks. General SMZ accumulation, growth performance, physiologic status, intestinal and hepatic health were systemically evaluated.

\section{Results}

The exposure experiment indicated that the SMZ accumulation in 0 . niloticus muscles, intestinal and aquaculture environment were positively related to the exposure dose. And withdrawal of antibiotics feed after 4th week, the SMZ residual in fish muscles, aquaculture water and sediment gradually decreased to safe level. LS and MS amount of antibiotics promoted the growth of fish accompanied by reduced feed coefficient in treated groups, while the fish growth in HS group was retarded. Exposure of $O$. niloticus to $\mathrm{SMZ}$ also increased the content of TG, promoted liver hypertrophy, and increased number of fat particles in liver. Antibiotics changed the content of intestinal short-chain fatty acids (SCFAs), and the changes in acetic acid were the most obvious. SMZ exposure reduced the biological diversity of the intestinal flora subsequently induced microbiota dysbiosis, and the degree was positively related to the exposure doses, and the experimental mainly inhibiting the growth of Fusobacteria.

\section{Conclusions}

Overall, exposure to of fish to legal doses of SMZ impair general physiological functions, intestinal flora and provokes health risk in fish. This study highlights the importance of rational and regulated use of $S M Z$ in aquaculture.

\section{Background}

Antibiotics poor absorption after medication, and overdose, coupled with their bioactivity and persistent behavior in global environment that has caused severe ecological sustainability and health risks [1-3], especially in fish, which also plays a vital role in human food safety. In aquaculture, fish cannot avoid exposure to antibiotics because of legal dietary medication to prevent and cure diseases $[4,5]$.

Sulfamethoxazole (SMZ) is bacteriostatic antibiotics used for treatments of bacterial diseases in cultured fish species, which blocks dihydrofolate intermediate production, thereby restricts the normal bacterial folic acid synthesis $[4,6]$. The SMZ is commonly administered at 100 to $200 \mathrm{mg} / \mathrm{kg}$ fish body weight per day for 5 days [7], depending on fish species, infection and country-specific legal requirements. Despite 
these legal directives, the antibiotics is used for long periods in aquaculture production [4], sometimes on daily basis [8]. Previous studies indicated that, SMZ is poorly absorbed in the guts of animals after medication, subsequently are excreted in urine and feces, either unchanged or modified into metabolites, which are transported into surface waters through runoff and subsurface drainage systems [9]. SMZ have been detected in freshwater at concentrations ranging from $259.60 \mathrm{ng} / \mathrm{L}$ to $385.00 \mathrm{ng} / \mathrm{L}$ [10]. These concentrations pose risks on aquatic species, raising global public concern on human health upon fish consumption $[6,11]$. However, no study has thoroughly evaluated the potential effects of using legal doses of SMZ in fish.

In this work, in order to deeply explore the differences of fish and the aquaculture environment, with the different SMZ addition set according to the aquaculture recommended doses. For this purpose, we exposed Nile tilapia (Oreochromis niloticus), a global economic, cultured and consumed species, to dietary treatments containing SMZ for 8 weeks (4 weeks with antibiotics fed and 4 weeks with normal fed, SCT 1084-2006 recommended the withdrawal period was 30 days). The present work aimed to explore the systemic effects of legal SMZ doses on antibiotic accumulation, general body functions, hepatic health and intestinal flora in fish. It provides a theoretical basis for the scientific use of SMZ in the aquaculture industry.

\section{Methods}

\section{Antibiotics and fish diet exposure}

Sulfamethoxazole (SMZ, purity $w>98.0 \%$ ) were purchased from ANPEL Laboratory Technologies Co., Ltd., Shanghai, China. About 400 O. niloticus fingerlings were supplied by fishery breeding base, the experiment was carried out in the ecological room of the Freshwater Fisheries Research Center of the Chinese Academy of Fishery Sciences. After acclimatization, all tested fingerlings were randomly distributed into 12 sterile 500 -litre tanks (30 fish per tank) filled with dechlorinated water to a volume of $400 \mathrm{~L}$ per tank.

Twenty-four hours before the experiment, the total initial mean weights of 0 . niloticus were determined as $16.33 \pm 0.50 \mathrm{~g}$ for all groups. In the experiment, refer to SCT 1084-2006 "Regulations for the use of sulfamethoxazole in aquaculture", considering the fluctuation of SMZ use in actual production, $O$. niloticus were exposed to three concentrations in feeds supplemented with the low-dose (LS, $20 \mathrm{mg} /(\mathrm{kg} \cdot \mathrm{d})$ ) and medium-dose (MS, SCT 1084-2006 recommended The therapeutic dose of $200 \mathrm{mg} /(\mathrm{kg} \cdot \mathrm{d}))$ and high dose (HS, 5 times the normal dose, $1000 \mathrm{mg} /(\mathrm{kg} \cdot \mathrm{d}))$. Convert it to the SMZ content in the feed as $0.67,6.67$ and $33.33 \mathrm{~g} / \mathrm{kg}$, respectively. A control tank was included in which $O$. niloticus were reared and treated similarly but deprived of antibiotics, each group set 3 parallel, total of 12 aquaculture tanks. All $O$. niloticus were hand-fed twice daily at 9:00 $\mathrm{h}$ and $15: 00 \mathrm{~h}$ and at $3 \%$ of their average body weight per day for 8 weeks ( 4 weeks with antibiotics fed and 4 weeks with blank fed). The entire breeding experiment does not change the water in order to maintain relatively stable concentrations 
as designed. The weight of individual 0 . niloticus was recorded weekly and the feed rations were adjusted accordingly.

\section{SMZ residual determination}

The 0 . niloticus muscles, intestinal contents and aquaculture water were collected at the beginning of the experiment, 4th, 6th and 8th weeks, the aquaculture sediment was collected at the end of the 8th week, the analysis of antibiotics concentrations was performed following $[12,13]$.

\section{Growth performance}

In the 8th week of the experiment, the 0 . niloticus were collected and their individual weights determined for weight gain and final weight estimations. The amount of feed and the weight of 0 . niloticus were used to calculate feed coefficient as the ratio of total wet weight gained by 0 . niloticus to total amount of feed fed in each group.

\section{Liver morphology and detection}

Randomly select 3 fish in each tank, MS-222 anesthesia was used to sample the fish and take the liver with dissection. The liver were washed with normal saline and fixed with $2.5 \%$ glutaraldehyde. The sections were stained according to the routine preparation procedures of transmission electron microscopy ultrathin section samples, which were to be observed, analyzed and photographed [14, 15]. Meanwhile, a reagent test kit was used to determine the triglyceride content (TG) of liver tissue.

\section{Short-chain fatty acid determination}

In the 4th week of the experiment, a $100 \mathrm{mg}$ of intestinal contents were collected from each breeding barrel for SCFAs, $100 \mu \mathrm{L}$ of $15 \%$ phosphoric acid, $50 \mu \mathrm{L}$ of $50 \mu \mathrm{g} / \mathrm{mL}$ of internal standard (isohexanoic acid) solution and $100 \mu \mathrm{L}$ of ether was added to homogenize for $1 \mathrm{~min}$, centrifuge at $4^{\circ} \mathrm{C}, 12000 \mathrm{rpm}$ for 10 min, the supernatant was analyzed by GC-MS, the detail instrument conditions was performed following ${ }^{[16]}$.

\section{Intestinal flora diversity determination}

In the 4th week of the experiment, the intestinal contents was quickly frozen in liquid nitrogen and stored at $-80^{\circ} \mathrm{C}$ for DNA extraction and $16 \mathrm{~s}$ rRNA (V3 + V4) high-throughput sequencing. After extracting the total DNA of the sample, primers 338F (5'-ACTCCTACGGGAGGCAGCAG-3') and 806R (5'-

GGACTACHVGGGTWTCTAAT-3') were designed according to the conserved region, and sequencing adapters were added to the ends of the primers to perform PCR amplification and the products purification, quantification, and homogenization are performed to form a sequencing library. The built library is first subjected to library quality inspection, and the qualified library is sequenced with Illumina HiSeq 2500. Filtered the sequencing data, and fed back the effective sequences after splicing. UCLUST (Edgar, 2002) in QIIME ${ }^{[17]}$ software was used to cluster Tags at a similarity level of 97\%, and obtain Operational Taxonomic Units (OTU), based on Silva (bacteria). Taxonomy database provides taxonomic annotations to OTU; Sobs, Ace, Chao1, Shannon, and Simpson indexes were used to compare the 
biological diversity within different treatment samples; PCoA analysis was used to compare the difference in the composition and structure of the biological community between different groups.

\section{Statistical analysis}

Isanger Shengxinyun was used to analyze and plots differences in bacterial flora structure. SPSS 25.0 software was used for significant difference analysis $(P<0.05$ indicates significant difference), all of the data are expressed as mean \pm SE $(n=3)$. Graphpad Prism 5.0, Adobe Illustrator and Origin 81 software ware used for mapping.

\section{Results And Discussion}

\section{SMZ residual characteristics}

In this study, the food intake of fish performed well, the feed coefficient at 4th week showed no significant difference. Various nutrients in the feed were digested and absorbed by the fish intestinal epithelial cells $[3,18]$. The feed ingredients also contained amount of SMZ $(0.019 \mu \mathrm{g} / \mathrm{kg})$. In NS group, the SMZ residual in fish muscles was below the maximum limited value (MRLs value, $100 \mu \mathrm{g} / \mathrm{kg}$ ) stipulated in the 235 announcement of the Ministry of Agriculture (Fig. 1a). And in other groups, the SMZ residual in fish muscles and intestinal contents reached the highest value in the 4th week, and the residual dose of SMZ in each group was closely related to the feed exposure (Fig. 1a, b). After returning to normal feed in the 6th -8th week, the residual SMZ in the fish intestinal contents were still at a high level, which may release into environment through fish excretion activity.

The SMZ residual in the aquaculture system mainly comes from the excretion of fish and the release of SMZ from the unconsumed bait [19]. The SMZ concentration in NS group were in the range of 0.14$1.31 \mu \mathrm{g} / \mathrm{L}$. And in other groups, the SMZ concentration increased rapidly from the start of aquaculture and reached a maximum in the 6th week, and then decreased rapidly, and the growth rate in the first 4 weeks was higher than that in the 4th to 6th weeks. At the 8th week, there was no difference in the residual of SMZ in the aquaculture waters of each group (Fig. 1c). These results shows that with SMZ feed, the SMZ residual in the aquaculture water will increase due to the presence of SMZ; after stopping $S M Z$ feed, the content of SMZ in the aquaculture water environment will decrease rapidly. The sediment located at the bottom of the culture system, and there is a large amount of fish excrement and bait accumulation [20]. And low oxygen and low temperature condition at bottom was not conducive to the degradation of SMZ [21]. Therefore, the SMZ residues are still at a high level at the end of aquaculture period. The SMZ residues in the sediments of each treatment group were also positively correlated with the amount of feed antibiotics.

\section{O. niloticus growth performance}

Growth performance is one of the most important aspects to fish farmers because it affects production and economic benefit in the aquaculture. LS and MS feeding group promoted growth of $O$. niloticus by 
reducind feed coefficient in LS and MS group (Fig. 2c). This result most likely occurred because the appropriate amount SMZ improved the activity of intestinal epithelial cells and promoted the digestion and absorption of nutrients [22, 23]. Antibiotics reduced the negative effects of anti-nutritional factors in feed. This finding agrees with those obtained in previous studies on an appropriate amount of antibiotics can promote the growth of farmed aquatic products [23-25]. For the exposure of 0 . niloticus to SMZ feed significantly retarded growth performance and weight gain in HS group $(P<0.05$; Fig. 2a, b, Table 1). This result is in line with excessive antibiotics may inhibit the growth of farmed aquatic products [26]. This finding may account for the excessive SMZ produced toxicity and impaired normal life activities of $O$. niloticus.

The liver is an accumulation organ for fish body fat. The fat in the fish liver mainly comes from the conversion and synthesis of excess protein, carbohydrates and absorption of fat in the feed [27]. The $O$. niloticus exposed to SMZ had fatter liver and higher viscera than control fish with the increased number of fat particles $(P<0.05 ; \mathrm{Fig} .3)$. These results indicated that, exposured to LS and MS concentration of SMZ have positive effects on fish growth performance, feed coefficient, except for HS diet. Exposure of $O$. niloticus to SMZ also increased the content of TG in liver $(P<0.05$; Fig. $2 \mathrm{~d})$. These finding agree with those obtained in previous studies on several animals including mice and rainbow trout [28,29], which showed that penicillin and erythromycin exposure greatly improved the fat accumulation may be limited fish energy metabolism and growth performance, this is most likely the reasons why the weight gain of $O$. niloticus in this experiment is LS, MS $>$ NS $>$ HS.

\section{The SCFAs contents}

SCFAs are the products of intestinal flora metabolizing and fermenting nutrients in feed. The contents of 7 SCFAs in the intestinal contents of $O$. niloticus in each treatment group were quantitatively analyzed. Only acetic acid, propionic acid, butyric acid, isovaleric acid, valeric acid and caproic acid were detected among all samples, isobutyric acid was not detected. The total content of 6 SCFAs in the intestinal contents of tilapia is between $45.11 \mu \mathrm{g} / \mathrm{g}-68.92 \mu \mathrm{g} / \mathrm{g}$ (Fig. 4a). And the percentage content of SCFAs in all samples are mainly acetic acid (accounting for $90.45 \%-94.44 \%$ ), propionic acid (2.88\%-4.38\%), butyric acid $(0.76 \%-1.49 \%)$, isovaleric acid $(0.31 \%-0.89 \%)$, valeric acid $(0.33 \%-0.42 \%)$ and caproic acid (0.65\%-3.15\%), respectively (Fig. 4b). In animal intestines, Bacteroides mainly produces acetic acid and propionic acid and Phylum Firmicutes can produce butyric acid [30], while acetic acid and propionic acid not only provide energy for cells but also participate in energy metabolism in the liver [31], and butyric acid can regenerate mucosal cells in the intestine [32,33]. The results of SCFAs showed that LS and MS diet would increase the content of SCFAs, and the main increase was propionic acid and butyric acid; HS group would reduce the content of SCFAs, and the main reduction was acetic acid. The content of SCFAs in the intestinal of $O$. niloticus in the SMZ treatment group was: LS, MS > NS > HS. These explanation supported the results that proper SMZ was fed to promote growth and excessive SMZ inhibited growth.

\section{Intestinal flora diversity}


The intestinal flora of 0 . niloticus has been established 20-60 days after hatching [34]. $16 \mathrm{~s}$ highthroughput sequencing was used to obtain bacterial data for each treatment group. Alpha diversity index analysis showed that all the community richness index (Sobs, Ace and Chao1) were decreased with the increase of SMZ dose, and the Sobs index in HS group was significantly lower than that of NS group $(P<$ 0.05). As for community evenness index (Shannon and Simpson), Shannon index diminished with increasing dose of SMZ, and Simpson index increased with increasing dose of SMZ. These results suggested that exposure to SMZ impair species abundance, biological abundance and intestinal flora biodiversity of 0 . niloticus (Table. 2). SMZ reduces the biodiversity of the intestinal flora, which was consistent with the results of other antibiotic feeding and breeding experiments [35]. PCoA was used to explore the similarity or difference of sample community composition. Based on the sample OUT level, the binary-jaccard was used to calculate the distance between the samples and to explore the influence of SMZ on the structure of the intestinal flora. The SMZ group (LS, MS, and HS) were significantly separated from the NS group (Fig. 5), the distance with NS group increased with the increase of the SMZ dose. These results indicated that the structure of intestines flora were altered in 0 . niloticus treated with SMZ feed.

\section{The intestinal flora composition and function}

Based on the annotation of OUT, the influence of SMZ on the intestinal flora composition and function from the perspective of community phylogeny was clarified, the species consumption of each group in the classification level (Phylum, class, and Genus) were shown in Fig. 6.

The main dominant species in the tested group were Fusobacteria, Proteobacteria, Cyanobacteria and Actinobacteria at the phylum level, which were basically consistent with the results of He's study [36], which indicating that SMZ reduced the biological diversity and changed the structure of the tilapia intestinal flora, and effected the number and proportion of dominant bacteria, but the main dominant bacteria at the phylum level was not changed. Fusobacteria in MS and HS group were much higher than NS and LS group, Proteobacteria in NS group was highest. Cyanobacteria in LS and MS group were higher than NS and HS group (Fig. 6a). These results implying that SMZ would affect the relative abundance of major dominant species.

The main dominant species were Fusobacteriia, Alphaproteobacteria, Cyanobacteria and Unclassified-pproteobacteria at the class level (Fig. 6b), and were Cetobacterium in Fusobacteria and norank-ccyanobacteria in Cyanobacteria at the genus level (Fig. 6c). These results showed that the abundance of Fusobacteria was positively correlated with the exposure dose. Species composition analysis showed that SMZ can inhibit the growth of Fusobacterium in the intestine of 0 . niloticus. Fusobacterium have obvious advantages in all samples, which may be related to their phylogenetic and phenotypic advantages [37]. Proper SMZ promotes its growth, which may be related to the large number of Fusobacterium and the characteristics of broad-spectrum antibacterial SMZ. The Cyanobacteria was main distributed in the aquaculture water, and the abundance was related to the nutrient content of the input (mainly feed) and the eutrophication of the aquaculture environment [38]. The Cyanobacteria in all 
tested tilapia intestine came from the same species; Actinomycetes are an important component of the freshwater plankton bacterial community [39], the Actinomycetes in the intestine of tilapia were also from the same species. This is most likely related to the culture environment (feed nutrition, SMZ and temperature). The intestinal flora is the most important group to maintain the intestinal microenvironment. The greater the number and uniformity of the intestinal flora, the stronger the resistance of the intestinal flora, micro ecology is also more stable [40]. Obviously, the addition of SMZ changed the balance of intestinal flora in $O$. niloticus.

\section{Conclusion}

The fish exposed to SMZ will cause the accumulation of antibiotics in organs and aquaculture environment. The LS and MS doses of SMZ will promote the growth (weight gain) of tilapia, except for HS group. This effect is that SMZ reduces the consumption of feed by reducing the biological diversity of the intestinal flora and induces intestinal flora produces more SCFAs, which in turn promotes the growth of $O$. niloticus; while HS group severely reduces the intestinal flora and affects the production of SCFAs, and also causes abnormal accumulation of fat in viscera, thereby inhibiting the growth of $O$. niloticus. Therefore, in the process of aquaculture, antibiotics should be used scientifically and controlled strictly.

\section{Abbreviations}

O.niloticus: Oreochromis niloticus; SMZ: Sulfamethoxazole; TG: Triglyceride content; SCFAs: Short-chain fatty acid; PCoA: Principal component analysis

\section{Declarations}

\section{Acknowledgements}

Not applicable.

\section{Authors' contributions}

Longxiang Fang, designed research, performed research, analyzed data; wrote the paper; Xi Chen, analyzed data; performed research, wrote the paper. Xiangbao Shan, performed research, analyzed data; Liping Qiu, performed research, analyzed data; Limin Fan, performed research, analyzed data; Shunlong Meng and Chao Song contributed funding; analyzed data; revised paper.

\section{Funding}

This work was supported by National Natural Science Foundation of China (31802271), and National Key R\&D Program of China (2017YFC1600704).

\section{Availability of data and materials}


All data generated or analyzed during this study were included in this published article.

\section{Ethics approval and consent to participate}

The procedures of the fish experiment in this work were approved by the Chinese Academy of Fishery Sciences.

\section{Consent for publication}

Not applicable.

\section{Competing interests}

The authors declare that they have no competing interests in this work.

\section{References}

1. Brandt KK, Amezquita A, Backhaus T, Boxall A, Coors A, Heberer T, et al. Ecotoxicological assessment of antibiotics: A call for improved consideration of microorganisms. Environ Int. 2015;85:189-205.

2. Carvalho IT, Santos L. Antibiotics in the aquatic environments: A review of the European scenario. Environ Int. 2016;94:736-57.

3. Limbu SM, Zhou L, Sun S, Zhang M, Du Z. Chronic exposure to low environmental concentrations and legal aquaculture doses of antibiotics cause systemic adverse effects in Nile tilapia and provoke differential human health risk. Environ Int. 2018;115:205-19.

4. Phu TM, Scippo ML, Phuong NT, Tien CTK, Son CH, Dalsgaard A. Withdrawal time for sulfamethoxazole and trimethoprim following treatment of striped catfish (Pangasianodon hypophthalmus) and hybrid red tilapia (Oreochromis mossambicus $\times$ Oreochromis niloticus). Aquaculture. 2015;437:256-62.

5. Zhang Q, Cheng J, Xin Q. Effects of tetracycline on developmental toxicity and molecular responses in zebrafish (Danio rerio) embryos. Ecotoxicology. 2015;24:707-19.

6. Johansson $\mathrm{CH}$, Janmar L, Backhaus T. Toxicity of ciprofloxacin and sulfamethoxazole to marine periphytic algae and bacteria. Aquat Toxicol. 2014;156:248-58.

7. Liu X, Steele JC, Meng XZ. Usage, residue, and human health risk of antibiotics in Chinese aquaculture: A review. Environ Pollut. 2017;223:161-9.

8. Pham DK, Chu J, Do NT, Brose F, Wertheim HFL. Monitoring antibiotic use and residue in freshwater aquaculture for domestic use in Vietnam. EcoHealth. 2015;12:480-9.

9. Zhang $Y$, Wang $X$, Yin X, Shi M, Dahlgren RA, Wang H. Toxicity assessment of combined fluoroquinolone and tetracycline exposure in zebrafish (Danio rerio). Environ Toxicol. 2016;31:736-50.

10. Chen K, Zhou JL. Occurrence and behavior of antibiotics in water and sediments from the Huangpu River, Shanghai, China. Chemosphere. 2014;95:604-12. 
11. Yan Z, Lu G, Ye Q, Liu J. Long-term effects of antibiotics, norfloxacin, and sulfamethoxazole, in a partial life-cycle study with zebrafish (Danio rerio): effects on growth, development, and reproduction. Environ Sci Pollut R. 2016;23:18222-8.

12. Song C, Li L, Zhang C, Kamira B, Qiu L, Fan L, et al. Occurrence and human dietary assessment of sulfonamide antibiotics in cultured fish around Tai Lake, China. Environ Sci Pollut Res. 2017a;24:17493-9.

13. Song C, Li L, Zhang C, Qiu L, Fan L, Wu W. Dietary risk ranking for residual antibiotics in cultured aquatic products around Tai Lake, China. Ecotox Environ Safe. 2017b;144:252-7.

14. Ribeiro HJ, Procópio MS, Gomes JMM, Vieira FO, Russo RC, Balzuweit K, et al. Functional dissimilarity of melanomacrophage centres in the liver and spleen from females of the teleost fish Prochilodus argenteus. Cell Tissue Res. 2011;346:417-25.

15. Samanta P, Bandyopadhyay N, Pal S, Mukherjee AK, Ghosh AR. Histopathological and ultramicroscopical changes in gill, liver and kidney of Anabas testudineus (Bloch) after chronic intoxication of almix (metsulfuron methyl 10.1\%+chlorimuron ethyl 10.1\%) herbicide. Ecotox Environ Safe. 2015;122:360-7.

16. Ebrahimi M, Daeman NH, Chong CM, Karami A, Kumar V, Hoseinifar SH, et al. Comparing the effects of different dietary organic acids on the growth, intestinal short-chain fatty acids, and liver histopathology of red hybrid tilapia (Oreochromis sp.) and potential use of these as preservatives. Fish Physiol Biochem. 2017;43:1195-207.

17. Capoaso J, Gregory KJ, Jesse S. QIIME allows analysis of high-throughput community sequencing data. Nat Methods. 2010;7:335-6.

18. Zhao H, Wang Y, Guo M, Mu MM, Yu H, Xing M. Grass carps co-exposed to environmentally relevant concentrations of cypermethrin and sulfamethoxazole bear immunodeficiency and are vulnerable to subsequent Aeromonas hydrophila infection. Environ Pollut. 2020;266:115156.

19. Xi X, Wang M, Chen Y, Yu S, Hong Y, Ma J, et al. Adaption of the microbial community to continuous exposures of multiple residual antibiotics in sediments from a salt-water aquacultural farm. $\mathrm{J}$ Hazard Mater. 2015;290:96-105.

20. Chen Y, Zhou JL, Cheng L, Zheng YY, Xu J. Sediment and salinity effects on the bioaccumulation of sulfamethoxazole in zebrafish (Danio rerio). Chemosphere. 2017;180:467-75.

21. Su T, Deng H, Benskin JP, Radke M. Biodegradation of sulfamethoxazole photo-transformation products in a water/sediment test. Chemosphere. 2016;148:518-25.

22. Dibner JJ, Richards JD. Antibiotic growth promoters in agriculture: history and mode of action. Poultry Sci. 2005;84:634-43.

23. Gaskins HR, Collier CT, Anderson DB. Antibiotics as growth promotants: mode of action. Anim Biotechnol. 2002;13:29-42.

24. Koh CB, Romano N, Zahrah AS, Ng WK. Effects of a dietary organic acids blend and oxytetracycline on the growth, nutrient utilization and total cultivable gut microbiota of the red hybrid tilapia,Oreochromissp., and resistance toStreptococcus agalactiae. Aquac Res. 2014;47:357-69. 
25. He S, Zhou Z, Meng K, Zhao H, Yao B, Ringo E, et al. Effects of dietary antibiotic growth promoter and Saccharomyces cerevisiae fermentation product on roduction, intestinal bacterial community, and nonspecific immunity of hybrid tilapia (Oreochromis niloticus female $\times$ Oreochromis aureus male). J Anim Sci. 2011;89:84-92.

26. Nakano T, Hayashi S, Nagamine N. Effect of excessive doses of oxytetracycline on stress-related biomarker expression in coho salmon. Environ Sci Pollut R. 2018;25:7121-8.

27. Chi L, Lai Y, Lai P, Liu C-W, Xue J, Ru H. Lipid and cholesterol homeostasis after arsenic exposure and antibiotic treatment in mice: potential role of the microbiota. Environ Health Persp. 2019;127:9700212.

28. Hu J, Luo H, Jiang Y, Chen P. Dietary capsaicin and antibiotics act synergistically to reduce nonalcoholic fatty liver disease induced by high fat diet in mice. Oncotarget. 2017;8:38161-75.

29. Rodrigues S, Antunes SC, Nunes B, Correia AT. Histological alterations in gills and liver of rainbow trout (Oncorhynchus mykiss) after exposure to the antibiotic oxytetracycline. Environ Toxicol Phar. 2017;53:164-76.

30. Morrison DJ, Preston T. Formation of short chain fatty acids by the gut microbiota and their impact on human metabolism. Gut Microbes. 2016;7:189-200.

31. Hong Y-H, Hong, Nishimura Y, Hishikawa D, Tsuzuki H, Tsuzuki, Miyahara H, Gotoh C, et al. Acetate and Propionate short chain fatty acids stimulate adipogenesis via GPCR43. Endocrinology. 2005;146:5092-9.

32. Karppinen S. Dietary fibre components of rye bran and their fermentation in vitro. Biotech Fac Sci U Helsinki. 2003;500:1-96.

33. Desai M, Seekatz A, Koropatkin N, Kamada N, Martens E. A dietary fiber-deprived gut microbiota degrades the colonic mucus barrier and enhances pathogen susceptibility. Cell. 2016;167:1339-53.

34. Sugita H, Fushino T, Oshima K, Deguchi Y. Microflora in the water and sediment of freshwater culture ponds. B Jpn Soc Sci Fisheries. 1985;51:91-7.

35. Viveros A, Chamorro S, Pizarro M, Arija I, Centeno C, Brenes A. Effects of dietary polyphenol-rich grape products on intestinal microflora and gut morphology in broiler chicks. Poultry Sci. 2011;90:566-78.

36. He S, Zhou Z, Liu Y, Cao Y, Meng K. Do dietary betaine and the antibiotic florfenicol influence the intestinal autochthonous bacterial community in hybrid tilapia (Oreochromis niloticus $\nabla \times 0$. aureus 凶)? World J Microb Biot. 2012;28:785-91.

37. Gupta SK, Raja SM, Gupta AB. Simultaneous nitrification-denitrification in a rotating biological contactor. Environ Technol. 1994;15:145-53.

38. Vasconcelos V. Eutrophicatton, toxic cyanobacteria and cyanotoxins: When ecosystems cry for help. Limnetica. 2006;25:425-32.

39. Allgaier M, Brückner S, Jaspers E, Grossart HP. Intra- and inter-lake variability of free-living and particle-associated Actinobacteria communities. Environ Microbiol. 2007;9:2728-41. 
40. Hooper LV, Macpherson AJ. Immune adaptations that maintain homeostasis with the intestinal microbiota. Nat Rev Immunol. 2010;10:159-69.

\section{Tables}

Due to technical limitations, table 1 is only available as a download in the Supplemental Files section.

\section{Figures}
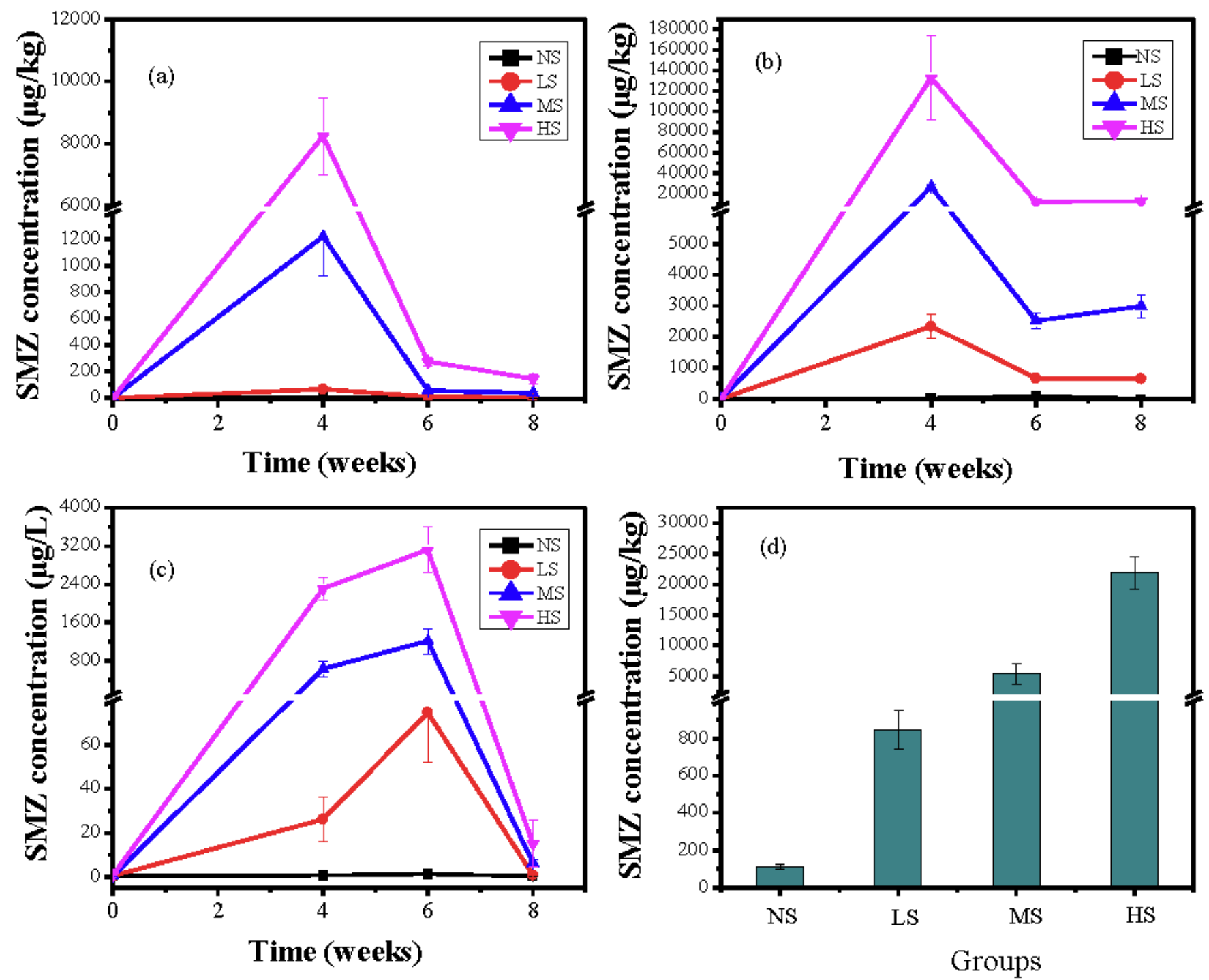

Figure 1

Dynamic changes of SMZ residues in O. niloticus. Note: (a) Muscles, (b) intestinal contents, (c) aquaculture water and (d) sediment under different doses of sulfamethoxazole feed. Values are means \pm 
SE $(n=20)$, the different number $(a, b)$ indicates significant difference between control and sulfamethoxazole diet $(P<0.05)$.
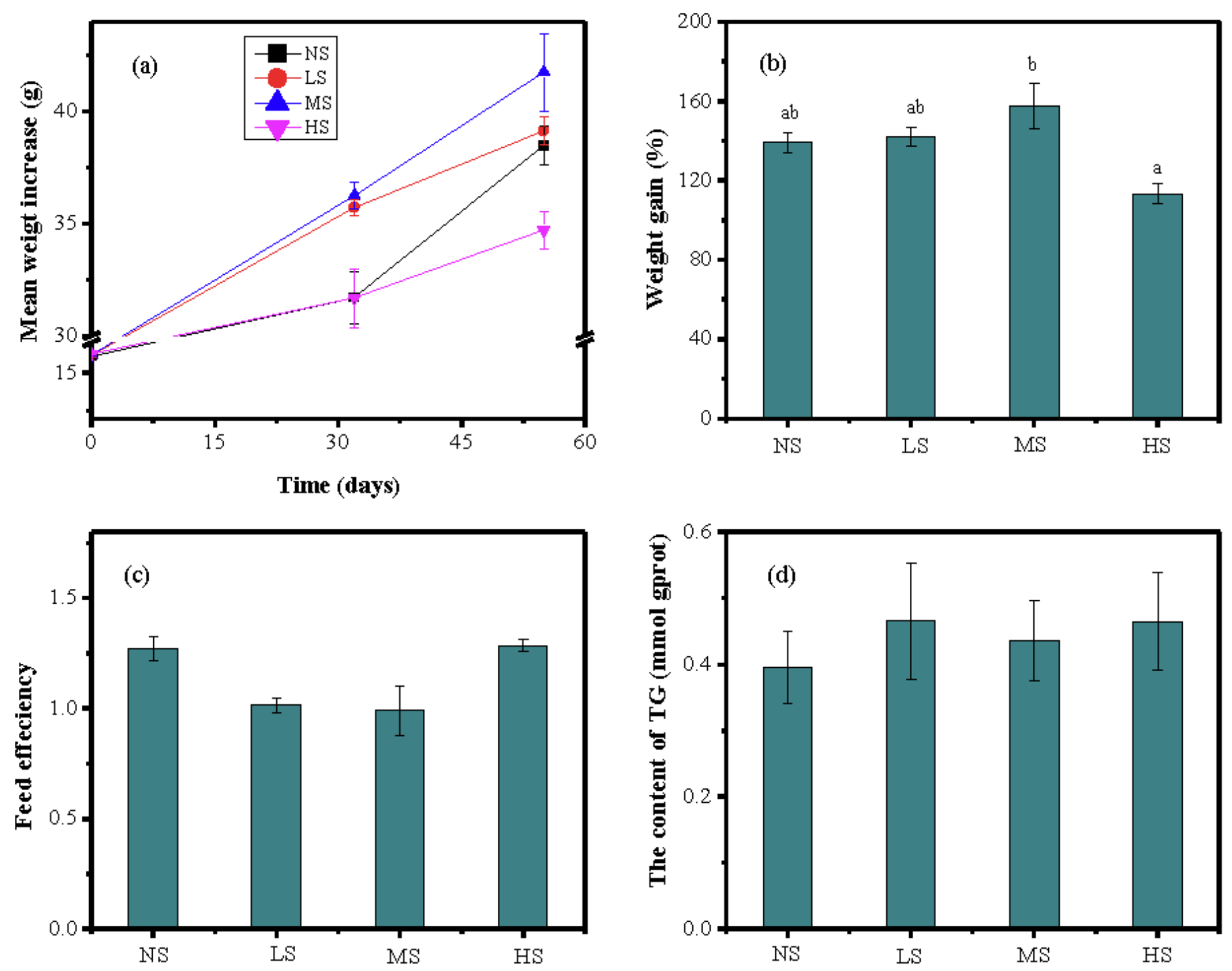

Figure 2

Growth performance of O. niloticus feed exposed to different doses of SMZ. Note: (a) Mean weight increase, (b) weight gain, (c) feed coefficient and (d) the content of triglyceride in fish liver. Values are means $\pm S E[n=30$ for $(a)$ and $(b)$ and $n=20$ for $(c)]$, the different number $(a, b)$ indicates significant difference between control and sulfamethoxazole diet group $(P<0.05)$. 

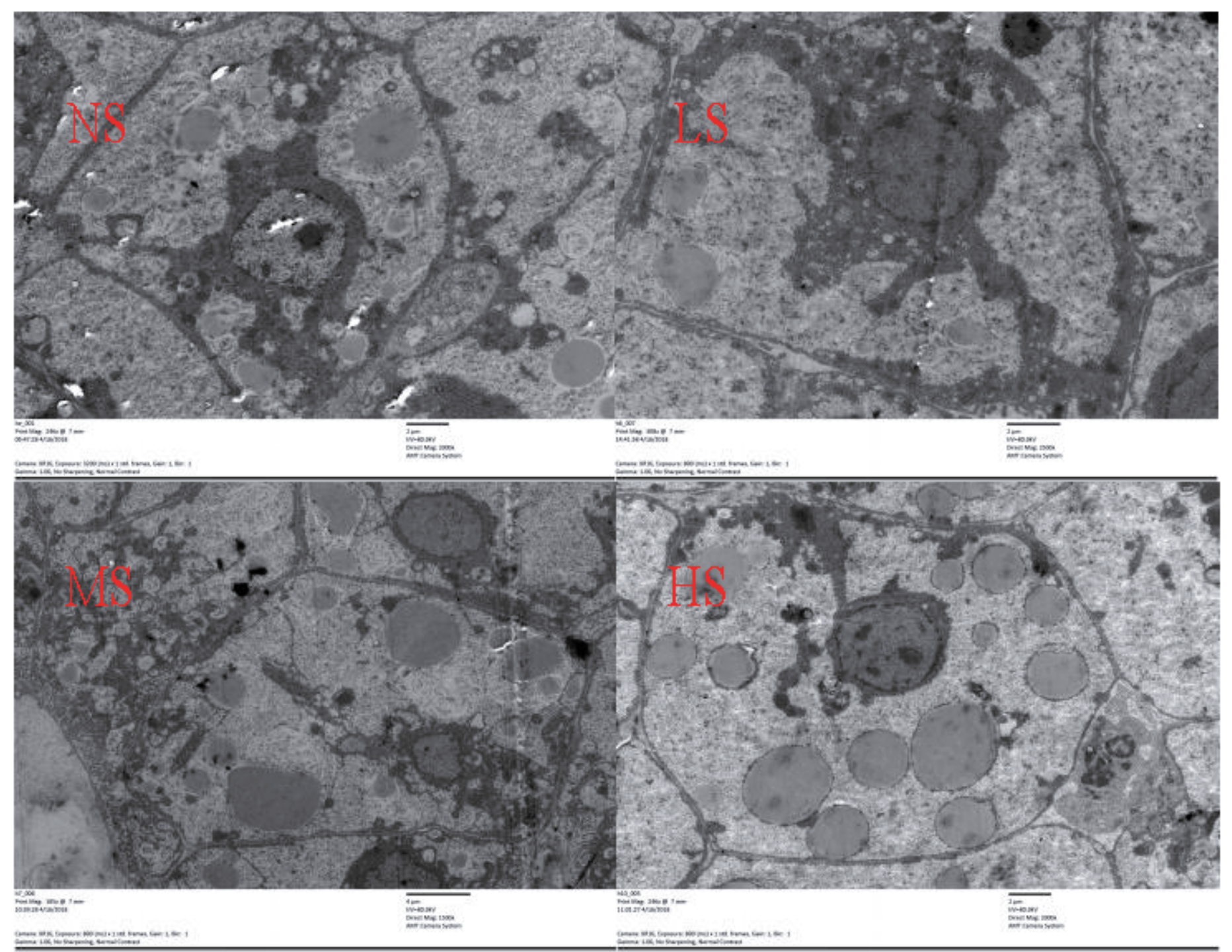

Figure 3

Fat accumulation in liver cells of 0 . niloticus in each culture group. 

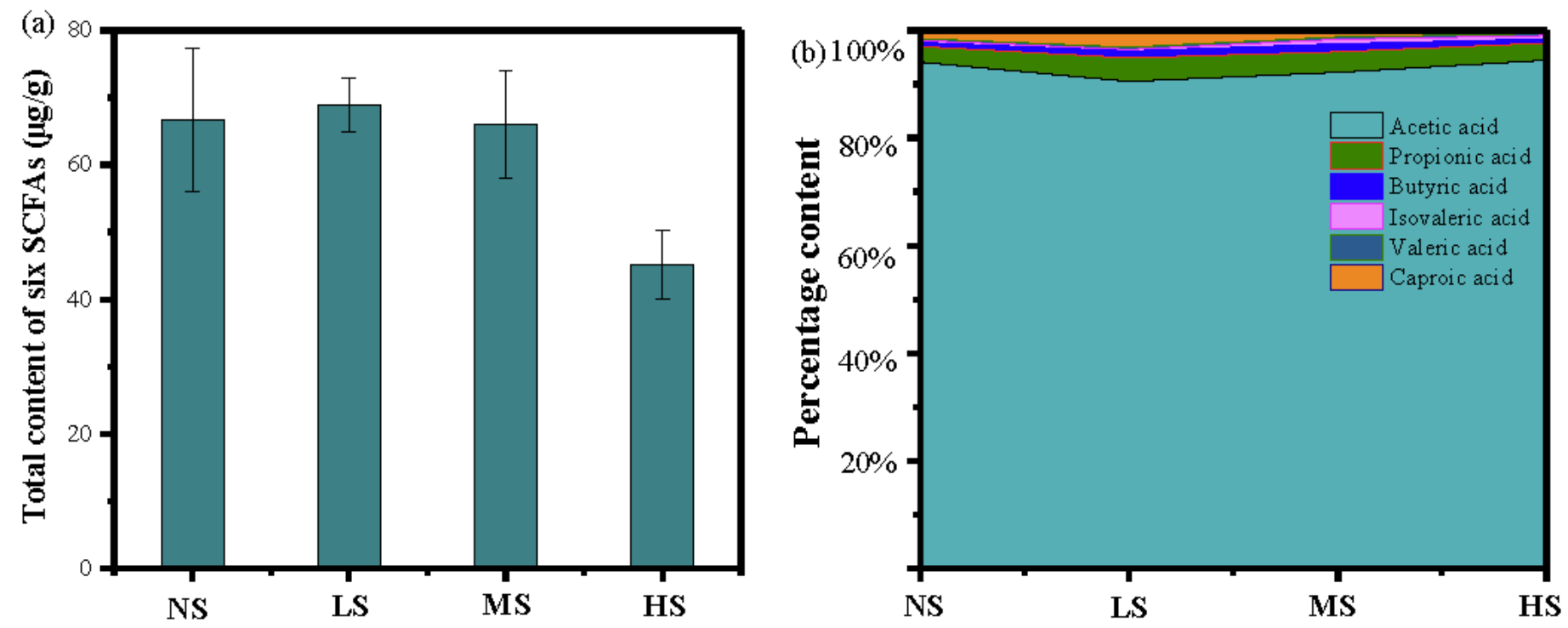

Figure 4

Intestinal SCFAs contents of O. niloticus feed and exposed to SMZ treatments. Note: (a) Total content of intestinal SCFAs, (b) the six of main composition of intestinal SCFAs, values are means \pm SE $(n=20)$. 


\section{PCOA on OTU level}

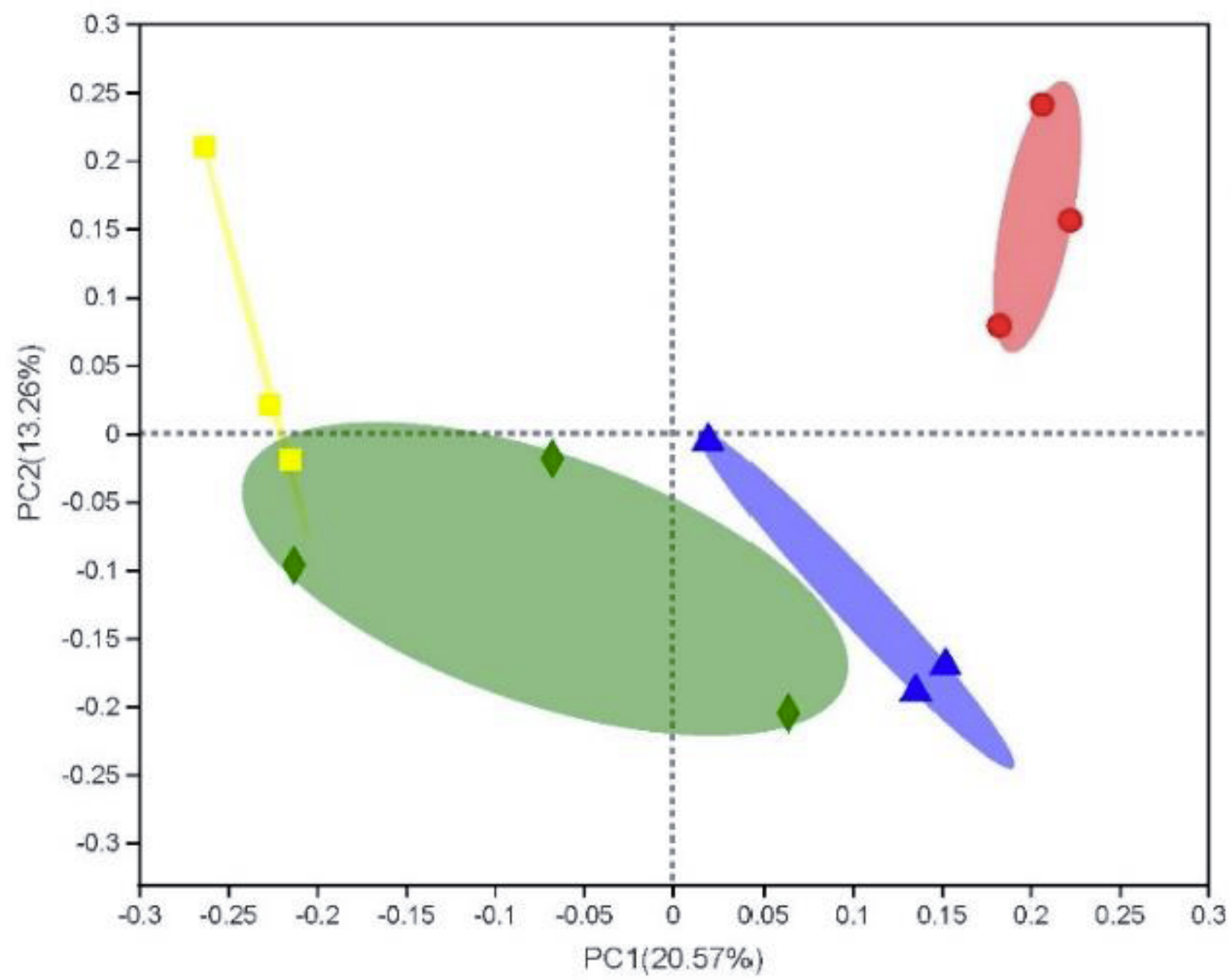

\section{Figure 5}

PCoA analysis of O. niloticus feed and exposed to SMZ treatments. 

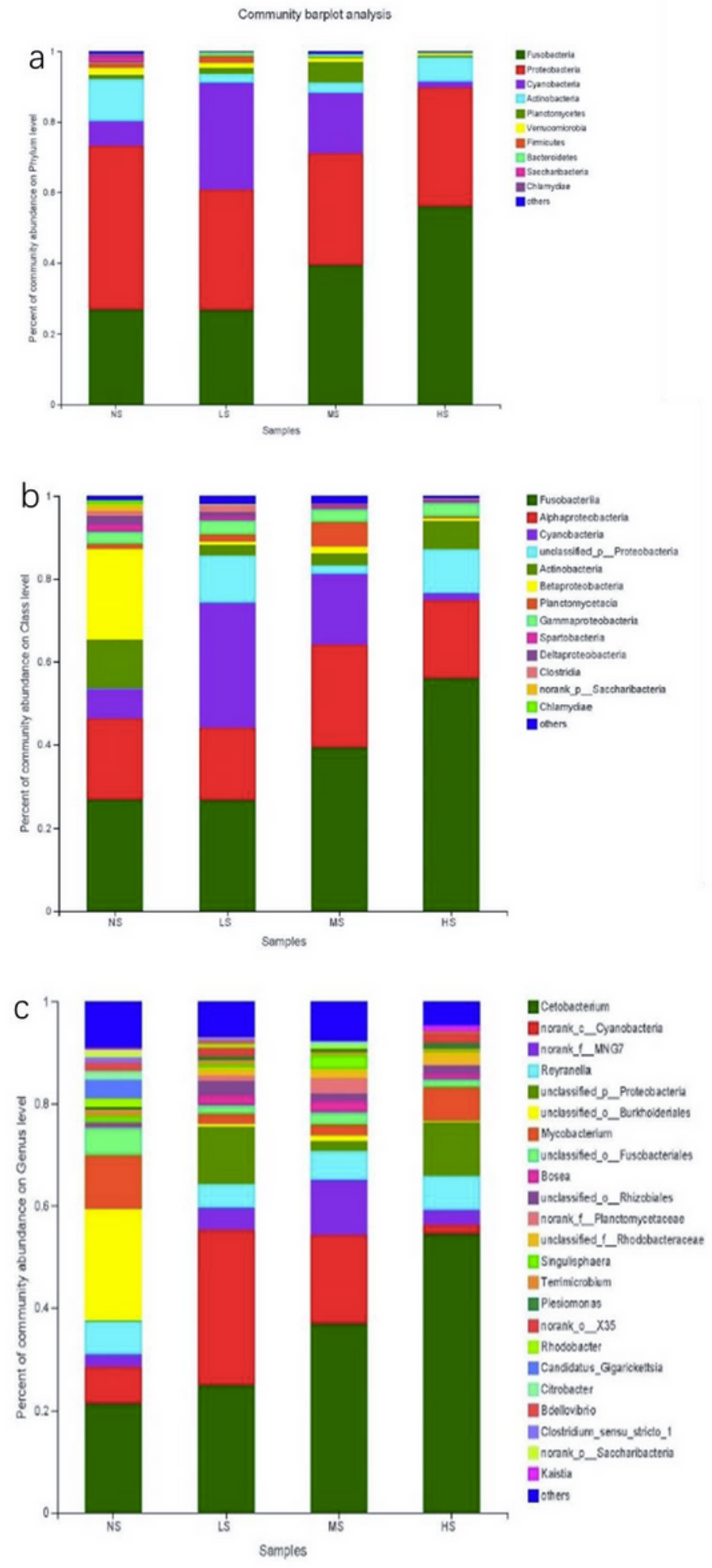

Figure 6

Effects of SMZ on species composition in O. niloticus intestinal folra. Note: (a) Phylum level, (b) class level and (c) genus level.

\section{Supplementary Files}


This is a list of supplementary files associated with this preprint. Click to download.

- Table.xlsx 